\title{
Investigation of Association between Slime Production by Candida Spp and Susceptibility to Fluconazole and Voriconazole
}

\author{
Banu Kaskatepe* and Sulhiye Yildiz \\ Ankara University, Faculty of Pharmacy, Department of Pharmaceutical Microbiology, Ankara, Turkey
}

*For correspondence: Email: bkaskatepe@ankara.edu.tr, Tel: +90 312 2033188; Fax: + 90312213181

Received: 17 November 2012

Revised accepted: 4 June 2013

\begin{abstract}
Purpose: To determine the susceptibilities of fluconazole and voriconazole based on slime production by Candida spp.

Methods: Candida strains (115) isolated in the period between January 2011 and January 2012 were included in this study. Conventional methods were used for the identification. Candida albicans and non-C. albicans isolates were tested for slime production with modified tube adherence test and antifungal resistance with disk diffusion method.

Results: Slime positivity was $31.3 \%$ in all Candida species. Slime positivity in non-C.albicans isolates (44.89\%) was higher than in C. albicans species (21.21\%). All C. albicans isolates were sensitive to fluconazole and voriconazole. The highest resistance to fluconazole (40\%) and voriconazole (5\%) was by C. glabrata strains.

Conclusion: Species definition and determination of antifungal susceptibility patterns are advised for the proper management and treatment of patients.
\end{abstract}

Keywords: Candida, Fluconazole, Voriconazole, Antifungal susceptibility, Slime

Tropical Journal of Pharmaceutical Research is indexed by Science Citation Index (SciSearch), Scopus, International Pharmaceutical Abstract, Chemical Abstracts, Embase, Index Copernicus, EBSCO, African Index Medicus, JournalSeek, Journal Citation Reports/Science Edition, Directory of Open Access Journals (DOAJ), African Journal Online, Bioline International, Open-J-Gate and Pharmacy Abstracts

\section{INTRODUCTION}

Candida spp. are members of normal flora and are also oppurtunistic pathogens that can cause serious systemic infections especially in immuncompromised patients. Candida infections have increased in the last two decades because of immunosuppresive treatments, long-term catheterisation, prolonged use of broad-spectrum antibiotics,cancer treatments and HIV infections. Various factors play a role in the pathogenesis of Candida infections, and slime production in Candida species is an important virulance factor which is associated with adherence to the surface of catheters and biomedical devices, and thus protects microorganisms from host defences. Slime (biofilm)-producing Candida species are known to be more resistant to immune response and antifungal agents which leads to treatment failure $[1,2]$.

The aim of this study was to determine the relationship between slime production by Candida spp and susceptibility to fluconazole and voriconazole. 


\section{EXPERIMENTAL}

\section{Media/chemical agents}

Sabouraud dextrose agar (SDA, Merck), Brain Heart Infision Broth (Plasmatec), Safranin (Merck) were used in this study.

\section{Strains}

A total of 115 Candida species, isolated from various clinical samples in the Clinical Microbiology Laboratory of Ankara Dışkapı Yıldırım Beyazıt Training Hospital, were included in this study. Candida species isolated from the same patient were excluded. Prior to being tested, all strains were subcultured twice on Sabouraud dextrose agar (SDA) to ensure viability and purity. For the identification of the isolates, conventional methods were used such as germ tube formation, microscopic morphology on cornmeal-Tween 80 Agar as well as commercial methods such as CHROMagar Candida. Candida dubliniensis isolates were identified on the basis of their initial dark green colony color on CHROMagar. If the species couldn't be identified by these methods they were classified as Candida spp. C.albicans ATCC 10231,reference strain was also included in this study.

\section{Slime production}

Slime production was determined using a modified tube adherence test. A loopful of organisms from the surface of a Sabouraud dextrose agar plate was inoculated into a polystyrene falcon tube containing $10 \mathrm{ml}$ of Sabouraud broth supplemented with glucose (final concentration, 8\%). The tubes were incubated at $35^{\circ} \mathrm{C}$ for $24 \mathrm{~h}$. The cell suspension in the tubes were poured out and washed with distilled water two times. After dying, $1 \%$ safranine, the tubes were examined for the presence of the viscid slime layer. Slime production by each isolate was scored as negative, weak positive $(1+)$, moderate positive $(2+$ or $3+)$ and strong positive(4+). Each isolate was tested at least three times and each tube was scored independently by two observers.

\section{Antifungal agents and susceptibility test}

Fluconazole (25 $\mu \mathrm{g}$, Oxoid) and voriconazole (1 $\mu \mathrm{g}$, Oxoid) disks were used for antifungal susceptibility tests. Antifungal susceptibility testing of Candida strains was performed according to the guidelines and criteria of Clinical and Laboratory Standards Institute (CLSI/M44-A) using disk diffusion method [3]. Inoculum was prepared from $24 \mathrm{~h}$ cultures in SDA. Plates containing Mueller-Hinton agar supplemented with $2 \%$ glucose and $0.5 \mu \mathrm{g} / \mathrm{ml}$ methylene blue at a depth of $4.0 \mathrm{~mm}$ were used. The agar surface was inoculated by using a swab dipped in a cell suspension adjusted to the turbidity of a 0.5 McFarland standard. Fluconazole and voriconazole disks were placed on the surfaces of the plates. After incubation at $35^{\circ} \mathrm{C}$ for $24 \mathrm{~h}$, the inhibition diameters around the disks were measured.

The interpretive criteria for the fluconazole and voriconazole disk diffusion tests were those of the CLSI: susceptible(S), zone diameters of $\geq 19$ $\mathrm{mm}$ fluconazole and $\geq 17 \mathrm{~mm}$ voriconazole; intermedier (I) zone diameters of 15 to $18 \mathrm{~mm}$ fluconazole and 14 to 16 voriconazole; and resistant (R), zone diameters of $\leq 14 \mathrm{~mm}$ fluconazole and $\leq 13 \mathrm{~mm}$ voriconazole.

\section{Statistical analysis}

The data were analyzed using SPSS program, version 17.0 and subjected to Chi-square test. At 95\% confidence interval, $P<0.05$ was considered statitically significant.

\section{RESULTS}

Slime activity and antifungal susceptibility of 115 Candida strains isolated from clinical samples were investigated in this study. The most common species recovered were $C$. albicans $(57.4 \%)$ followed by C. glabrata (17.4\%), C.tropicalis (12.2\%), C. parapsilosis (5.21\%), C. dubliniensis $(5.21 \%)$ and Candida spp. (2.60 \%) A total of $36(31.3 \%)$ out of 115 Candida isolates were slime producers. The highest slime production was found among $C$. parapsilosis isolates $(66.66 \%)$. It was also shown that nonC. albicans strains $(44.89 \%)$ produced significantly higher slime factor than $C$. albicans strains $(21.21 \%)(p=0.007)$. Slime production results are reported in Table 1.

A total of eight C.glabrata isolates were resistant to fluconazole and one C.glabrata isolate was intermedier (I) to this antifungal. All Candida isolates were susceptible to voriconazole except one resistant and one intermedier C. glabrata isolates. These isolates were also resistant to fluconazole. Antifungal susceptibility data are listed in Table 2. 
Table 1: Slime production by Candida isolates

\begin{tabular}{lccccc}
\hline Yeast (n) & Negative & $\begin{array}{c}\text { Weak } \\
\text { positive }\end{array}$ & $\begin{array}{c}\text { Moderate } \\
\text { positive }\end{array}$ & $\begin{array}{c}\text { Strong } \\
\text { positive }\end{array}$ & Total \\
\hline C. albicans (66) & 52 & 5 & 1 & 8 & 14 \\
C. glabrata (20) & 15 & 4 & 1 & - & 5 \\
C. tropicalis (14) & 5 & - & 1 & 8 & 9 \\
C. parapsilosis (6) & 2 & - & - & 4 & 4 \\
C. dubliniensis (6) & 4 & - & 2 & - & 2 \\
Candida spp.(3) & 1 & - & 1 & 1 & 2 \\
Total non-C.albicans (49) & 27 & 4 & 5 & 13 & 22 \\
\hline
\end{tabular}

Table 2: Antifungal susceptibility of Candida spp.

\begin{tabular}{lcccccc}
\hline \multirow{2}{*}{ Species } & \multicolumn{2}{c}{$\mathbf{S} \mathbf{n}(\%)$} & \multicolumn{2}{c}{$\mathbf{I} \mathbf{n}(\%)$} & \multicolumn{2}{c}{$\mathbf{R} \mathbf{n}(\%)$} \\
\cline { 2 - 7 } & Flu & Vor & Flu & Vor & Flu & Vor \\
\hline C. albicans & $66(100)$ & $66(100)$ & - & - & - & - \\
C. glabrata & $11(55)$ & $18(90)$ & $1(5)$ & $1(5)$ & $8(40)$ & $1(5)$ \\
C. tropicalis & $14(100)$ & $14(100)$ & - & - & - & - \\
C. parapsilosis & $6(100)$ & $6(100)$ & - & - & - & - \\
C. dubliniensis & $3(50)$ & $6(100)$ & $3(50)$ & - & - & - \\
Non-C. albicans & $37(75.5)$ & $47(96)$ & $4(8.2)$ & $1(2)$ & $8(16.3)$ & $1(2)$ \\
Candida spp. & $3(100)$ & $3(100)$ & - & - & - & - \\
Total & $103(89.6)$ & $113(98.2)$ & $4(3.4)$ & $1(0.9)$ & $8(7)$ & $1(0.9)$ \\
\hline
\end{tabular}

We investigated the correlation between slime activity and antifungal susceptibility of the two antifungal agents. For all Candida species no correlation was detected between slime and antifungal susceptibility.

\section{DISCUSSION}

Candida spp. can cause both superficial and serious systemic diseases and are now recognized as one of the major agents of nosocomial infections. Recent data from the US National Nosocomial Infections Surveillance System [1] rank these organisms as the fourth most common cause of bloodstream infection. Biofilms are the structured microbial communities that are attached and encased in the matrix of exopolimeric material and are important for the development of clinical infection. Many candida infections involve the formation of biofilms on implanted devices. When bacteria exist in the biofilm form they are $10-1000$ times more resistant to antibiotics than are planktonic cells $[1,4]$. In the present study, we investigated the correlation between slime production and resistance to two antifungal agents.

Various rates of slime production have been reported in a number of studies. While Mohandas and Ballal reported high rates of 51 and $90.32 \%$ in 2011 for C. albicans and non-C. albicans isolates, respectively [5], in other studies which were supported by our results as well, lower resistance rates were found [6,7]. In this study total slime positivity rate was $31.31 \%$
(21.21\% in C. albicans and $44.89 \%$ in non-C. albicans isolates).

Similarly to our results, more recent studies showed slime production is common especially in non-C. albicans strains. In Tumbarello et. al study, they reported $22.6 \%$ slime positivity rate in C. albicans and $33.3 \%$ in non-C. albicans isolates [6]. Yildırım et. al found $17 \%$ in $C$. albicans, $33 \%$ in non- $C$. albicans [7]. In contrast to this findings, Dag et al found slime positivity 39.3 and $37.7 \%$ in C. albicans and non-C. albicans, respectively [8]. We found that there was a statistically significantly higher slime production in non-C. albicans strains than in $C$. albicans isolates. The highest slime production was found in $C$. tropicalis among all non- $C$. albicans isolates including $C$. glabrata, $C$. parapsilosis, C. dubliniensis.

Despite the widespread use of fluconazole for more than two decades, we found no evidence that C. albicans has developed increased resistance to fluconazole. All $C$. albicans species were sensitive to both antifungal agents. However, resistance rates to fluconazole in $C$. albicans are different in the other studies [9-11]. It is known that non-C. albicans species increase in candida infections and these species have a resistance to antifungal drugs. Non-C. albicans species have various degrees of susceptibility to the frequently used antifungal drugs while $C$. krusei is intrinsically resistant to fluconazole, $C$. glabrata is less susceptible or has higher minimal inhibitory concentrations (MICs) than other Candida species [9] C. glabrata is an 
oppurtunistic pathogen that has become increasingly frequent in bloodstream and mucosal infections in immunocompromised patients.The increasing use of azole antifungals for the treatment of $C$. glabrata infections has resulted in emergence of resistance strains [11]. In the present study, there was a statistically significant higher fluconazole resistance in nonC. albicans than C. albicans isolates $(p=0.005)$. Resistance to fluconazole was observed relatively high, mainly in isolates of $C$. glabrata. Eight $(40 \%)$ C. glabrata isolates were found resistant to fluconazole and one C. glabrata was found intermedier. The resistance rates for fluconazole in C. glabrata are varried in the other studies.

All Candida spp. were susceptible to voriconazole except one C. glabrata strain which was also resistant to fluconazole. In addition, one C. glabrata isolate was found intermedier and it was also resistance to fluconozole too. Cross resistance between fluconazole and voriconazole is described among isolates of C.glabrata. The results of our antifungal susceptibility test are generally consistent with the findings from other studies. Similarly in Gültekin et al's study conducted with 46 Candida spp isolated from blood samples, all Candida isolates were determined to susceptible to fluconazole and voriconazole [12]. However, rates of resistance tofluconazole $(5 \%)$ and voriconazole $(7.7 \%)$ have been reported for $C$. albicans isolates [10].

In the present study which was conducted with 66 C. albicans and 49 non-C. albicans strains, we investigated the association between slime activity and susceptibility to fluconazole and variconazole. The results showed that some Candida species produced slime but the antifungal susceptibility test performed indicate that some of these species were susceptible to fluconazole and voriconazole. For C. albicans and non-C. albicans, no correlation was detected between slime activity and the susceptibility of the two antifungal agents because all $C$. albicans, C. tropicalis and C. parapsilosis strains were sensitive to both antifungal agents. Similar results have been found in other studies In one of them, conducted with $19 \mathrm{C}$. parapsilosis and $35 \mathrm{C}$. albicans strains, the authors investigated whether slime activity patterns correlated with the strains' susceptibility to fluconazole, ketoconazole and amphothericine B. They did not find correlation between slime activity and the MIC of all three antifungal agents [13]. Also, Shin et al did not find any significant association between biofilm production and clinical characteristics of candidemia due to $C$. albicans, since only two of 30 blood isolates of C.albicans in their study were biofilm-positive [2]. Yücesoy et al investigated the biofilm production of various Candida strains with tube adherence method and compared this activity with fluconazole and amphotericin B susceptibility. They found no statistically significant difference between biofilm activity and susceptibility to amphotericin B. However, statistically significant difference was found between biofilm activity and susceptibility to fluconazole $(p=0.03)$ [9]. These divergent results underlines the need for further studies.

\section{CONCLUSION}

Candida spp. isolated from various clinical samples were highly susceptible to the tested antifungals, namely, fluconazole and voriconazole. Since voriconazole exhibits higher efficacy than fluconazole in non-C. albicans isolates it may be appropriate to prefer voriconazole in the treatment of fungal infections caused by fluconazole resistant non-C. albicans strains.

\section{REFERENCES}

1. Douglas LJ. Candida biofilms and their role in infection. Trends Microbiol 2003; 11: 30-36

2. Shin JH., Kee SJ., Shin G., Kim H., Shin DH, Lee SK., Suh $S P$. ., Ryang DW.Biofilm production by isolates of Candida species recovered from nonneutropenic patients: comparison of bloodstream isolates with isolates from other sources. J Clin Microbiol 2002; 40: 1244-1248

3. CLSI. Method for Antifungal Disk Diffusion Susceptibility Testing of Yeasts: Approved Guideline. M44-A, Wayne, PA: CLSI, 2004

4. Ramage $G$, Rajendran R, Sherry L, Williams C. Fungal biofilm resistance Int J Microbiol 2012; doi: 10. 1155/2012/52821

5. Vinitha $M$, Ballal M. Distribution of Candida species in different clinical samples and their virulence: Biofilm formation, proteinase and phospholipase production: $A$ study on hospitalized patients in Southern India. J Global Infect Dis 2011; 3: 4-8

6. Tumbarello M., Posteraro B., Trecarichi EM., Fiori B., Rossi M., Porta R., Donati KG., Sorda M., Spanu T., Fadda G., Cauda R., Sanguinetti M. Biofilm production by Candida Species and Inadequate antifungal therapy as predictors of mortality for patients with candidemia. J Clin Microbiol 2007; 45: 1843-1850

7. Yıldırım M., Mumcuoğlu I, Kursun Ş., Koldas K., Yetener V., Balaban N. Comparison of certain virulence factors in Candida albicans and non-albicans candida strains isolated as infectious agent. Türk Mikrobiyol Cem Derg. 2009; 39:62-68

8. Dag I, Kiraz N, Oz Y. Evaluation of different detection methods of biofilm formation in clinical Candida isolates. Afr J Microbiol Res. 2010; 4(24): 27632768

9. Yücesoy $M$, Karaman M. Biofilm production and antifungal susceptibility patterns of Candida species. Mikrobiyol Bült. 2004; 38: 91-98.

10. Basma R, Barada G, Ojaimi N, Khalaf RA. Susceptibility of Candida albicans to common and novel antifungal drugs, and relationship between the mating type locus and resistance, in Lebanese hospital isolates. Mycoses 2008; 52: 141-148.

Trop J Pharm Res, October 2013;12 (5): 824 
11. Pfaller MA, Diekema DJ, Gibbs DL. Results from the ARTEMIS disk global antifungal surveillance study, 1997 to 2007: a 10,5- year analysis of susceptibilities of Candida species to fluconazole and voriconazole as determined by CLS standardized disk diffusion. J Clin Microbiol 2010, 48: 1366-1377

12. Gultekin B, Eyigör M, Tiryaki Y, Kırdar S, Aydın N. Investigation of antifungal susceptibilities and some virulence factors of Candida strains isolated from blood cultures and genotyping by RAPD-PCR. Mikrobiyol Bült 2011; 45: 306-317

13. Ozkan S, Kaynak F, Kalkancı A, Abbasoğlu U, Kustimur $S$. Slime production and proteinase activity of Candida species isolated from blood samples and comparison of these activities with minimum inhibitory concentration values of antifungal agents. Mem Inst Oswaldo Cruz 2005; 100: 319-324 